\title{
An Experimental Investigation of Nanoparticles Assisted Surfactant Flooding for Improving Oil Recovery in a Micromodel System
}

\author{
Elias Khalafi ${ }^{1}$, Abdolnabi Hashemi ${ }^{1}$, Mehdi Zallaghi' ${ }^{2 *}$ and Riyaz Kharrat ${ }^{2}$ \\ ${ }^{1}$ Department of Petroleum Engineering, Petroleum University of Technology, Ahwaz, Iran \\ ${ }^{2}$ Tehran Petroleum Research Center, Petroleum University of Technology, Tehran, Iran
}

\begin{abstract}
The mechanisms involved in nanoparticle application in enhanced oil recovery processes particularly when nanoparticles are used in conjunction with other chemical agents are still controversial. In this study, the main focus is on pore scale investigation of nanoparticle-surfactant mixture flooding as an enhanced oil recovery process. Five spot glass micro model experiments were conducted to study the oil recovery mechanisms in the presence of hydrophilic silica nanoparticles at various concentrations. Macroscopic oil recovery as well as pore fluid distributions were evaluated by the continuous images taken from the micro model during the injection process. The results represent that wettability alteration is the most important factor contributing to additional oil recovery when nanoparticles exist in the injected solution. Nanoparticles could significantly improve the oil recovery obtained during water and surfactant flooding. The oil film on the pore walls were slightly thinned by the sole surfactant solution while with the addition of nanoparticles, it was completely removed and became strongly water wet surface.
\end{abstract}

Keywords: Nanoparticle; EOR; Micro model; Surfactant flooding; Wettability alteration

\section{Inroduction}

Nanotechnology in the petroleum industry has gained enormous interest in the recent years, which is reflected in the amount of literature available. Nanoparticles for EOR purposes seem gradually to become the cutting-edge technology the ability of nanoparticles involves altering certain factors of the formation and oil properties which can be taken as an advantage of them in enhance oil recovery scenarios. This involves introducing these nanoparticles into formations and studying are effect on oil recovery.

Wettability is a one of the key factors which affects oil recovery from reservoir rocks. It extremely affects the flow priority and also distribution of fluids through porous medium. Changing wettability towards adequate circumstances (more water preferable) to increas oil recovery factor has been selected in plenty of research studies. These works show the high capability of surfactants to change wetness of surface [1-5]. Other associated mechanism with surfactant injection is the interfacial tension reduction between oil and water owing to mitigate capillary forces which allows recovering of trapped oil. Several researches have focused on obtain ultra-low IFT values using surfactants to increase its effectiveness [6-8]. Surfactant injection is characterized by Critical Micelle Concentration (CMC) and solubilization ratio parameters which are essential to Optimum design of surfactant flooding program [9].

Addition of alkali (e.g. sodium carbonate) reduces the adsorption of surfactant on rock surface which is the crucial factor for the process to applicable it for carbonate reservoirs. Thus, ultra IFT and wettability modification make desirable conditions for improve oil recovery form oil-wet carbonate rocks as well [10]. The best surfactant formulation is function of phase behavior, good solubility and low loss of surfactant in formation. Besides, more successful surfactant flooding relies on ultra IFT at optimum salinity and low retention [11].

Micromodels are two-dimensional structure of the porous medium which uses to visualize the flow behavior at pore scale. It can be used for observation of flow and transport phenomena at the microscale during fluid displacement in EOR process [12,13].
Pore structure has known as an effective parameter during fluid displacement; therefore, many works are done with the purpose of investigating pore morphology using micromodel. These studies show that the shape of pores and their connection to others has influenced on flow direction and ultimate oil recovery $[5,14,15]$.

Application of nanoparticle in enhanced oil recovery has drawn attentions to petroleum industry over the last decade. Experimental work at lab scale on different rock types, sandstone and carbonate, to investigate underling mechanisms have been subject of many researches. Several mechanisms have been proposed for as follows: wettability alteration, reduction of interfacial tension, oil viscosity and mobility ratio reduction [16-18]. Nanofluid flooding under secondary and tertiary injection schemes in Berea sandstone core plugs increases recovery $8 \%$ and $2 \%$ of OOIP respectively [17]. Also, nanoparticles have been used as an agent to stabilize oil/water emulsions and foam to improve the mobility control in EOR process [19-22]. Some researchers applied nanoparticles to improve surfactant functions such as their ability of IFT reduction [23-25] and decreasing surfactant adsorption [26].

Surfactant injection EOR has confirmed via a wide variety of examinations form lab to field application. Much research has been done in this case of individual application of surfactant and nanomaterial as EOR agent. However, based on literature survey, it is concluded that a few work has been done to evaluate the feasibility of using combination of nanoparticles and surfactant in EOR processes by means of micro-model.

"Corresponding author: Zallaghi M, Tehran Petroleum Research Center, Petroleum University of Technology, Tehran, Iran, Tel: 00989375168295; E-mail: m.zallaghi@put.ac.ir

Received November 08, 2017; Accepted February 01, 2018; Published February 07, 2018

Citation: Khalafi E, Hashemi A, Zallaghi M, Kharrat R (2018) An Experimenta Investigation of Nanoparticles Assisted Surfactant Flooding for Improving Oil Recovery in a Micromodel System. J Pet Environ Biotechnol 9: 355. doi: 10.4172/2157-7463.1000355

Copyright: $\odot 2018$ Khalafi E, et al. This is an open-access article distributed unde the terms of the Creative Commons Attribution License, which permits unrestricted use, distribution, and reproduction in any medium, provided the original author and source are credited. 
The objectives of this study are to carry out a systematic experiment on the effect of adding nanoparticles in surfactant solution on increasing oil recovery. Thus, several injection tests into micromodel were designed and performed. During the processes a series of photos were captured in order to observe oil displacement by injected fluid [27].

\section{Research Methodology}

\section{Experimental procedure}

Experiments were carried out in a one-quarter five spot etched glass micromodel. The micromodel apparatus involving a high-pressure air cylinder, precise flow rate pump (Quizix QL-700 with an injection accuracy of $10^{-5} \mathrm{ml} / \mathrm{min}$ ), transfer vessels, high resolution NIKON D-900 camera, digital microscope, micromodel holder and glass micromodel. Figure 1 shows a schematic of the micromodel system. The micromodels glass plate has approximately $60 \mathrm{~mm}$ length, $60 \mathrm{~mm}$ width. Figure 2 represents a glass micromodel pattern that used in this set of experiments. The Table 1 summarizes physical properties of the models. Before running each test, the glass micromodel was cleaned to assure no oil is remained after previous run. During cleaning procedure, distilled water and toluene were injected into micromodel to circulation with several cycles.

To change the natural water-wet surface of glass micromodel to oil-wet condition, a multi-step procedure called "Silinization" applied in which a chemical material "TriChloroMethylSilane" employed. The procedure is as follows:

- Rinse micromodel with common existing solvents in laboratory like toluene.

- Rinse micromodel with distilled water.

- Rinse micromodel with $\mathrm{HCl}$ for at least 15 minutes.

- Rinse glass surface with distilled water then dry it for at least 30 $\min$ at $150^{\circ} \mathrm{C}$ in oven.

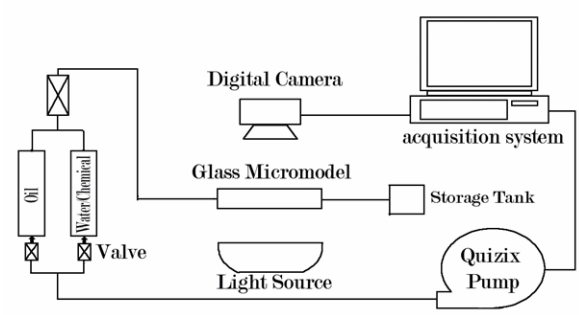

Figure 1: Micromodel system.

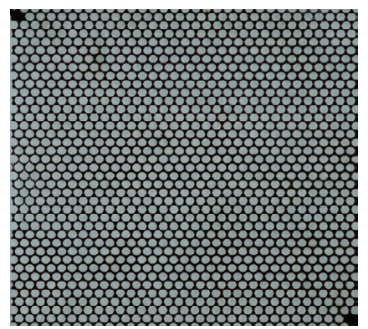

Figure 2: Schematic of homogenous glass micromodel.

\begin{tabular}{|c|c|c|c|c|}
\hline Model type & $\begin{array}{c}\text { Throat } \\
\text { diameter }(\boldsymbol{\mu m})\end{array}$ & $\begin{array}{c}\text { Etched } \\
\text { depth }(\boldsymbol{\mu m})\end{array}$ & $\begin{array}{c}\text { Permeability } \\
(\mathbf{m D})\end{array}$ & Porosity (\%) \\
\hline Homogeneous & 200 & 65 & 1100 & 37 \\
\hline \multicolumn{4}{|c}{ Table 1: Summary of physical properties. }
\end{tabular}

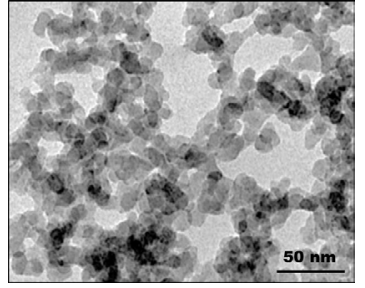

Figure 3: TEM image of silica nanoparticles used.

- Prepare a dilute solution of $98 \%$ dehydrate toluene and $2 \%$ TriChloroMethylSilane.

- Rinse glass surface with dilute solution for at least 20 min then rinse it with methanol.

- Dry micromodel at $100^{\circ} \mathrm{C}$ in oven for at least $1 \mathrm{hr}$.

Experiments started with saturation of micromodel with water and crude oil respectively. This process aims to establish initial condition of micromodel. Then chemical flooding takes place to displace oil as an EOR agent. During injection of desired solution, a series of photos were captured to observe the process of displacing oil with injected fluid. Preparation of both models, homogeneous and fractured model, before flooding was the same.

\section{Materials}

This section contains the information about the solutions used in the experiments and describes the procedure followed in micromodel injection processes. All the experiments were performed using 10000 ppm $\mathrm{NaCl}$ brine and a reservoir oil with viscosity of $14 c p$ at ambient condition. The crude oil was obtained from an Iranian oil field having an API of 21 degrees. Chemical materials including silica nanoparticle and Sodium Dodecyl Benzene sulfonate (SDBS) surfactant. The nanoparticles were ordered from Evonik Industries and consist of Hydrophilic Fumed Silica (AEROSIL 300) with purity of 99.8\%. The specific surface area is $300 \pm 30 \mathrm{~m}^{2} / \mathrm{g}$ and the average particle diameter is $7 \mathrm{~nm}$. It should be noticed that nanoparticles are approximately spherical in shape as observed in TEM images represented in Figure 3.

SDBS is an anionic surfactant which used in many purpose in oil laboratory due to its capability in lowering IFT and changing wettability of surface. The aqueous surfactant solutions obtained by adding adequate amount of SDBS into distilled water. Brine solution of 10000 ppm NACL which was prepared by solution of adequate amount of NACL salt provided by MERK Company into distilled water was used as connate water.

Conductivity measurement tool was used to determine critical micelle concentration (CMC). After preparation of injection solation, a series of flooding experiments were performed in micromodel apparatus at ambient pressure and temperature.

The values of sweep efficiency during these experiments were obtained by pixel analyzing of captured images after sharpening images using graphically software, Photoshop. To show the performance of displacing process and its capability of recovering inaccessible oil after breakthrough, the values of oil recoveries reported at the time of breakthrough and after 1.2 PV of injection. Composition of injected fluids during chemical injection is listed in Table 1. Experiments include water flooding; surfactant flooding, nanoparticle solution flooding, and nanoparticles assisted surfactant flooding are designed to examine research targets. 


\section{Results and Discussion}

\section{CMC determination}

$\mathrm{CMC}$ values were measured at $0 \mathrm{ppm}$ and $10000 \mathrm{ppm}$ of $\mathrm{NaCl}$ salinity to be $2240 \mathrm{ppm}$ and $700 \mathrm{ppm}$, respectively. Then silica nanoparticles with $1000 \mathrm{ppm}$ were added to the solution which results in reduction of initial CMC values to 1700 and $600 \mathrm{ppm}$, respectively.

\section{Static tests}

To evaluate the ability of emulsion formation several test tubes with different concentrations were investigated. Fig 4 shows emulsion formation using nanoparticles (a), surfactant (b), and Nano-surfactant (c). Observation shows that nanoparticles assisted surfactant have more ability to establish emulsion.

\section{Micromodel results}

This part presents results of various sets of experiments of chemical flooding that can improve oil recovery at pore scale. Experiments include water flooding, surfactant flooding, nanofluid flooding, and nanofluid assisted surfactant flooding which were conducted under different scenarios, as mentioned in Table 2.

Water flooding (base case): Water flooding considered as base case to comparison of various experiments strategy which results in oil recovery of $36 \%$. Water crossed the pattern during water flooding in a nearly straight path resulted a significant amount of oil behind as shown Figure 4. Scanning pore walls using digital microscope after water flooding shows that no wettability alternation has occurred during water flooding shown in Figure 5.

Microscopic flow phenomena observed during brine injection in shown in Figure 6. The wall of pore throat coated with thin film of oil which represents oil-wet condition that result in low oil recovery.

\begin{tabular}{|c|c|c|c|}
\hline Case \# & SDBS $\mathbf{( p p m )}$ & $\mathbf{S i O}_{\mathbf{2}} \mathbf{( p p m )}$ & Nacl $\mathbf{p p m})$ \\
\hline 1 & 200 & 0 & 10000 \\
\hline 2 & 500 & 0 & 10000 \\
\hline 3 & 1000 & 0 & 10000 \\
\hline 4 & 2000 & 0 & 10000 \\
\hline 5 & 0 & 200 & 10000 \\
\hline 6 & 0 & 500 & 10000 \\
\hline 7 & 0 & 1000 & 10000 \\
\hline 8 & 0 & 2000 & 10000 \\
\hline 9 & 500 & 200 & 10000 \\
\hline 10 & 500 & 1000 & 10000 \\
\hline 11 & 500 & 2000 & 10000 \\
\hline 12 & 1000 & 200 & 10000 \\
\hline 13 & 1000 & 1000 & 10000 \\
\hline 14 & 1000 & 2000 & 10000 \\
\hline
\end{tabular}

Table 2: Illustrates composition of the injected fluids.

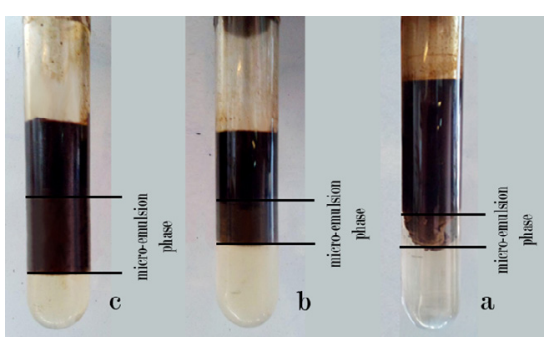

Figure 4: Emulsion formation in test tube.

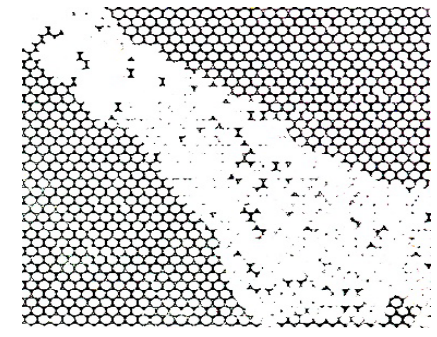

Figure 5: Flow path of displacing water.

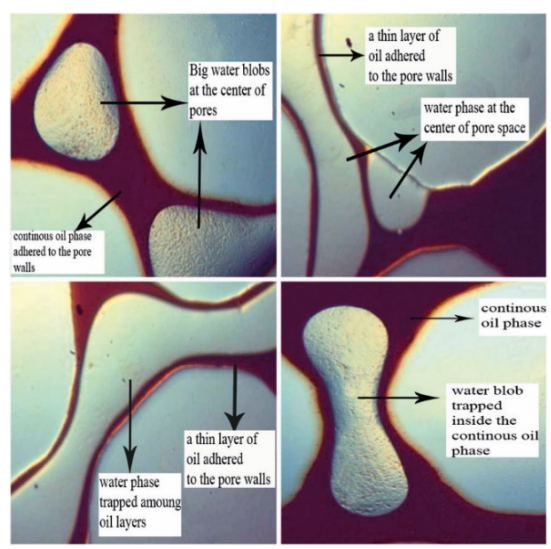

Figure 6: Initial oil wet condition of pore walls.

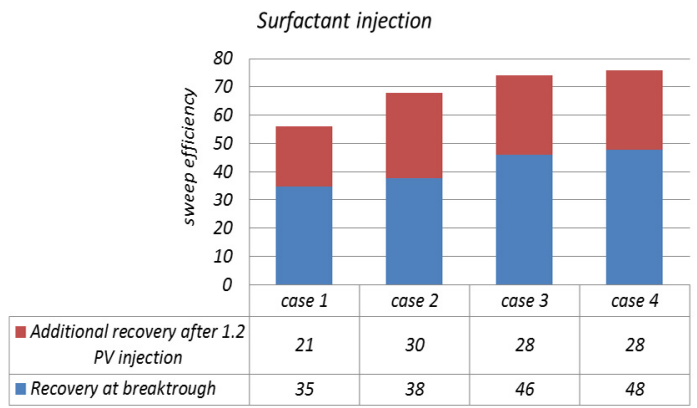

Figure 7: Values of areal sweep efficiency during injection of surfactant solution in micromodel.

Surfactant flooding: In this strategy, a series of surfactant flooding processes, as expressed in Table 2, were performed. Figure 7 shows the results of oil recovery when only surfactant was injected.

These 4 cases can be classified in 2 categories, namely surfactant concentrations below and above CMC. In low surfactant concentrations (below $\mathrm{CMC}$ ), increasing surfactant concentration resulted in more oil recovery. This increment in recovery was more pronounced after occurring surfactant solution breakthrough. When surfactant concentration was more than $\mathrm{CMC}$, oil recovery didn't change significantly with increasing concentration of surfactant.

After breakthrough, addition of oil recovery mainly depends on the value of interfacial tension and rheology of injected fluid. With respect to the behavior of IFT below and upper than CMC, it can be concluded that in low concentration, IFT had an extreme effect on sweeping of oil that was inaccessible before breakthrough. If surfactant concentration is more enough than CMC, then surfactant concentration has not any 
influence on IFT reduction. Therefore, oil recovery cannot be changed significantly.

Nanofluid flooding: In the case of nanofluid flooding, nanofluid solution with concentration of $200,500,1000$, and 2000 were injected in micromodel. According to the results nanofluid injection can improve oil recovery upto $70 \%$.

\section{Nanoparticle assisted surfactant flooding}

The effect of nanoparticles on assisting surfactant flooding for incremental oil recovery was investigated by performing six sets of experiments, as mentioned in Table 2 cases 9 to 14 .

Oil recovery was increased up to $73 \%$ when nanoparticle and surfactant were injected simultaneously. Figure 8 shows the values of oil recovery when nanoparticles were injected as a solution independently. In this set of runs, it has been observed that with increasing nanoparticle concentrations, oil recovery will be increased at breakthrough time dramatically. After reaching breakthrough, oil recovery did not change significantly with increasing particles concentration (Figure 9).

From analyzing captured images during injection, the main reason of nanofluid capability in enhancing oil recovery was thought as changing in flow path after injecting a fraction of pore volume.

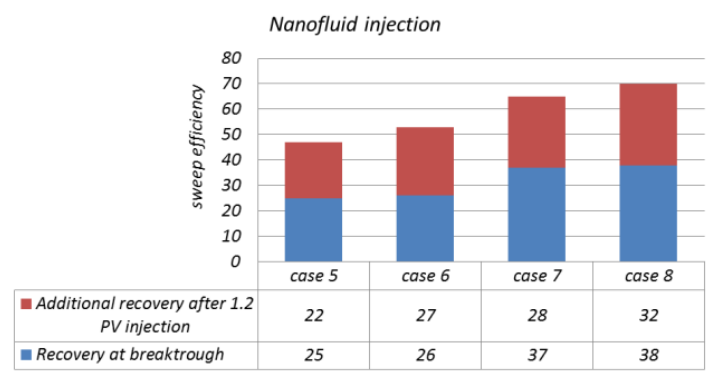

Figure 8: Results of nanofluid in micromodel.

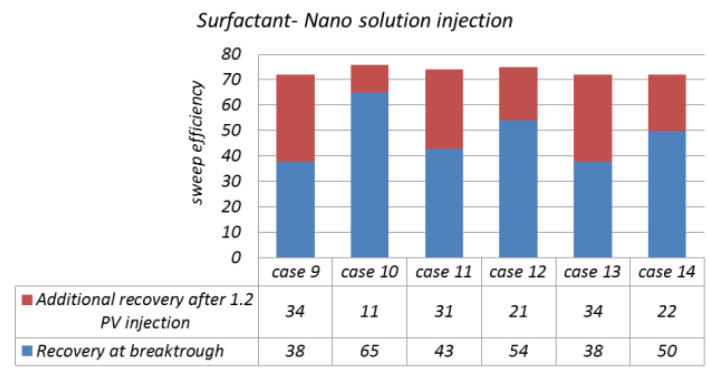

Figure 9: Values of areal sweep efficiency during injection of surfactant-nano solution in micromodel.

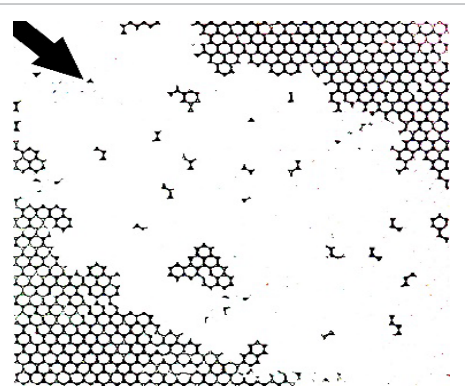

Figure 10: Flow path of displacing surfactant solution (1000 ppm).

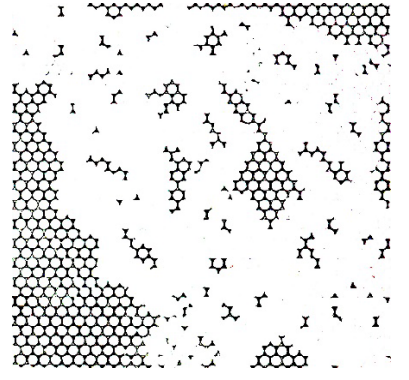

Figure 11: Flow path of displacing nanoparticle solution (2000 ppm).

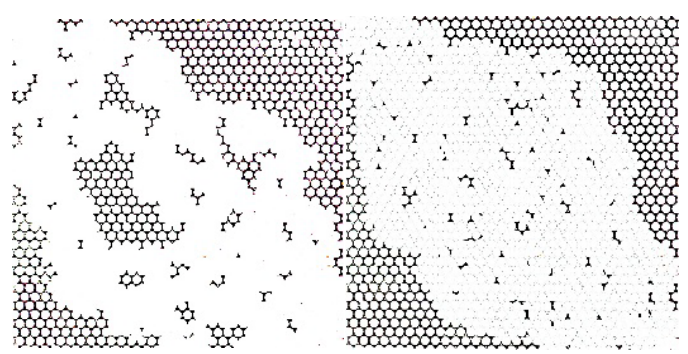

Figure 12: a) Injection of nanofluid (1000 ppm). b) Injection of surfactant-nano solution

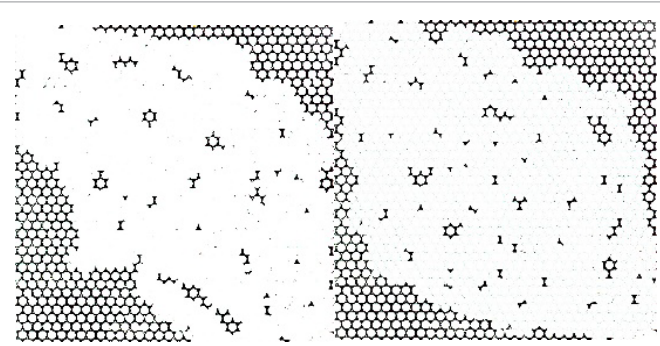

Figure 13: a) Injection of surfactant solution. b) Injection of surfactant-nano solution.

This might be due to change of pore walls wetness. In other words, nanoparticles alter oil wet condition of pore walls to water wet and consequently change the flow path.

When nanoparticles assisted surfactant flooding oil recovery increased dramatically in comparison with water injection. At the time of breakthrough, the solution with lowest concentration of surfactant and the optimum nanoparticles concentration (1000 ppm) showed highest value of recovery. In all cases, recovery value's after 1.2 PVs of injected solution were nearly at the same level.

\section{Comparison of the results}

Figures 10-13 shows a comparison illustration of the flow path in cases of water, surfactant, nanoparticles and surfactant-nanoparticles injection. Addition of surfactant into the injecting water has changed the flow path and displacing process performance extremely (Figure 10). In this case, swept area covered a large portion of entire model and, except some large oil blobs; the swept area was free of oil after flooding. In the case of nanoparticle injection, swept area was extended but, many discontinuous oil blobs have been observed after process (Figure 11). In this case, it seems that nanoparticles have changed the flow path by altering wettability. When nanoparticles were added into the surfactant solution, efficiency was improved significantly (Figures 12 


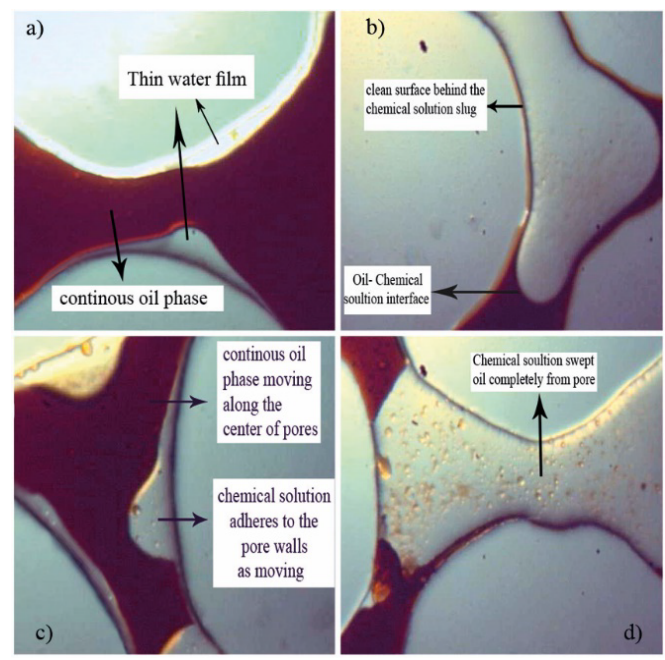

Figure 14: Change in wetness of pore walls after injection of chemical solution.

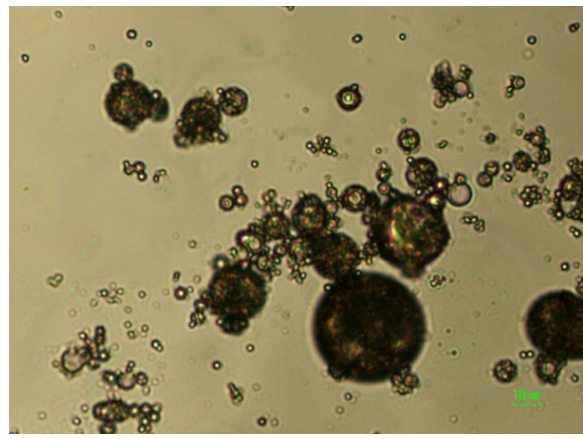

Figure 15: Emulsion formation in micromodel.

and 13). Also, the pattern of swept area has been changed in respect to the cases of water and surfactant injection. Presence of both surfactant and nanoparticles in injection fluid has decreased the remained oil blobs in swept area. In the following figures, the impact of nanoparticles on performance of surfactant solution is obviously clear. The effect of silica nanoparticles and surfactant solutions on fluid distribution on the walls and throats has investigated by several microscopic pictures using digital microscope.

Figures $14 \mathrm{a}$ and $14 \mathrm{~b}$ shows initial wetness of pore walls before flooding. A thin layer of oil adheres to pore walls in the presence of connate water that dictates oil wet condition. After running chemical fluid, digital photos were taken to confirm ability of chemical agents in changing wettability. Figures $14 \mathrm{c}$ and $14 \mathrm{~d}$ illustrate wetness of pore walls after chemical flooding. In these cases, the ability of surfactantnanoparticle augmented fluid in changing wettability is shown. This change in wettability was more visible at high concentration of nanoparticles.

Also, microscope images of micromodel confirm oil-in-water emulsion formation during nanoparticle assisted surfactant flooding in micromodel system, as shown in Figure 15.

Emulsions increase the viscosity of displacing fluid which results in improving the mobility ratio and leads to a better sweep efficiency. Emulsions increase viscosity of displacing fluid which results in improving the mobility ratio and leads to a better sweep efficiency. Aqueous dispersion of nanoparticles with surfactant enhanced emulsions formation and stabilization due to their surface activities. Therefore, injection fluid viscosity enhanced and provide better conformance which is important in macroscopic recovery efficiency.

\section{Conclusion}

This set of experiments provides considerable insight into the mechanisms of oil recovery by nanoparticles assisted surfactant solutions in porous medium of micromodel. Consequently, the following conclusions are based on the qualitative results:

1) Nanoparticle assisted surfactant flooding increases areal oil sweep efficiency. In this process nano-surfactant fluid will sweep the area which was bypassed during the water injection.

2) Direct observation of pore walls shows change in wettability as a result of using surfactant and nanoparticles. Injected water into the glass micromodel moves from inlet port of micromodel to the outlet port in a nearly linear path, resulting considerable amount of oil recovery after flooding.

3) By comparing recovery values at breakthrough and after 1.2 PV of injection fluid, it can be concluded that augmented surfactantparticles solution yields more oil recovery with respect to separately nanoparticle and surfactant solution at high enough concentrations.

4) Hydrophilic silica nanoparticle can extremely change the wettability of oil wet surface to water wet.

5) Remaining oil blob sizes after flooding in case of nanofluid injection was fairly large. Adding nanoparticle to surfactant solution not only has decreased the size but also yield cleaner area at swept region.

6) The interaction of matrix and fracture became higher than before, when a surfactant was added into the flooding fluid in existence of chemical agent.

7) In both test tubes and micro models, emulsion formation was observed during nano-surfactant assistant flooding which led to improvement in sweep efficiency.

\section{Acknowledgment}

The authors would like to thank the technical support of Tehran Petroleum Research Center-PUT.

\section{References}

1. Morrow NR (1990) Wettability and its effect on oil recovery. SPE-21621-PA Journal of Petroleum Technology.

2. Vijapurapu CS, Rao DN (2003) Effect of brine dilution and surfactant concentration on spreading and wettability. SPE international symposium on oilfield chemistry. Houston, Texas, USA.

3. Wu Y, Shuler PJ, Blanco M, Tang Y, Goddard WA (2006) A study of wetting behavior and surfactant EOR in carbonates with model compounds. SPE/DOE symposium on improved oil recovery. Tulsa, Oklahoma, USA.

4. Seethepalli A, Adibhatla B, Mohanty KK (2004) Physicochemical interactions during surfactant flooding of fractured carbonate reservoirs. Soc Pet Eng J 2: 619-626.

5. Jamaloei B, Kharrat R (2010)Analysis of microscopic displacement mechanisms of dilute surfactant flooding in oil-wet and water-wet porous media. J Transp Porous Med 81: 1-19.

6. Wade WH, Morgan JC, Schechter RS, Jacobson JK, Salager JL (1978) Interfacial tension and phase behavior of surfactant systems. Soc Pet Eng J 18: 242-252.

7. Glover CJ, Puerto MC, Maerker JM, Sandvik EL (1979) Surfactant phase behavior and retention in porous media. Soc Pet Eng J 19: 183-193. 
Citation: Khalafi E, Hashemi A, Zallaghi M, Kharrat R (2018) An Experimental Investigation of Nanoparticles Assisted Surfactant Flooding for Improving Oil Recovery in a Micromodel System. J Pet Environ Biotechnol 9: 355. doi: 10.4172/2157-7463.1000355

Page 6 of 6

8. Yadali Jamaloei B, Kharrat R (2010) Analysis of microscopic displacement mechanisms of dilute surfactant flooding in oil-wet and water-wet porous media. Transp Porous Media 81: 1-19.

9. Sheng JJ (2013) Review of surfactant enhanced oil recovery in carbonate reservoirs. Advances in Petroleum Exploration and Development 6: 1-10.

10. Hirasaki GJ, Miller CA, Puerto M (2011) Recent advances in surfactant EOR. SPE-115386-PA. SPE Journal.

11. Spildo K, Sun L, Djurhuus K, Skauge A (2014) A strategy for low cost, effective surfactant injection. Journal of Petroleum Science and Engineering 117: 8-14.

12. Wan J, Tetsu S, Tokunaga TK, Tsang CF, Bodvarsson GS (1996) Improved glass micromodel methods for studies of flow and transport in fractured porous media authors. Water Resources Research 32: 1955-1964.

13. Corapcioglu YM, Chowdhury S, Sharon E (1997) Micromodel visualization and quantification of solute transport in porous media. Water Resources Research 33: $2547-2558$

14. Yadali Jamaloei B, Kharrat $R$ (2011) The influence of pore geometry on flow instability and pore-scale displacement mechanisms of dilute surfactant flooding in mixed wet porous media. J Porous Media 14: 91-105.

15. Dullien FAL, Dhawan GK, Gurak N, Babjak L (1972) A relationship between pore structure and residual oil saturation in tertiary surfactant floods. Soc Pet Eng J 12: 289-296.

16. Ogolo NA, Olafuyi OA Onyekonwu MO (2012) Enhanced oil recovery using nanoparticles. SPE-160847-MS. SPE Saudi Arabia Section Technical Symposium and Exhibition, Al-Khobar, Saudi Arabia.

17. Torsater O, Engeset B, Hendraningrat L, Suwarno S (2012) Improved oi recovery by nanofluids flooding: An experimental study. SPE-163335-MS. SPE Kuwait International Petroleum Conference and Exhibition, 10-12 December, Kuwait City, Kuwait.

18. Roustaei A, Bagherzadeh H (2015) Experimental investigation of $\mathrm{SiO}_{2}$ nanoparticles on enhanced oil recovery of carbonate reservoirs.J Petrol Explor Prod Technol 5: 27.

19. Zhang T, Roberts MR, Bryant SL, Huh C (2009) Foams and emulsions stabilized with nanoparticles for potential conformance control applications. Paper SPE 121744, presented at the 2009, SPE International Symposium on Oilfield Chemistry, The Woodlands, Texas, USA.

20. Zhang T, Davidson A, Bryant SL, Huh C (2010) Nanoparticle-stabilized emulsions for applications in enhanced oil recovery. In: SPE improved oil recovery symposium, Tulsa, OK, USA.

21. Pei HH, Zhang GC, Ge JJ, Zhang J, Zhang Q, et al. (2015) Investigation of nanoparticle and surfactant stabilized emulsion to enhance oil recovery in waterflooded heavy oil reservoirs. Paper SPE-174488-MS, presented at the Canada Heavy Oil Technical Conference, Calgary, Alberta, Canada.

22. Kim I, Worthen AJ, Lotfollahi M, Johnston KP, DiCarlo DA, et al. (2016) Nanoparticle-Stabilized Emulsions for improved mobility control for adversemobility waterflooding. Paper SPE-179644-MS, presented at the SPE Improved Oil Recovery Conference, Tulsa, Oklahoma, USA.

23. Suleimanov BA, Ismailov FS, Veliyev EF (2011) Nanofluid for enhanced oil recovery. J Pet Sci Eng 78: 431-437.

24. Ma H, Luo M, Dai L (2008) Influences of surfactant and nanoparticle assembly on effective interfacial tensions. Phys. Chem. Chem Phys 10: 2207-2213.

25. Moghadam TF, Azizian S (2014) Effect of ZnO nanoparticles on the interfacial behavior of anionic surfactant at liquid/liquid interfaces. Colloids Surf A 457: 333-339.

26. Esmaeilzadeh P, Bahramian A, Fakhroueian Z (2011) Adsorption of anionic cationic and nonionic surfactants on carbonate rock in presence of $\mathrm{ZrO}_{2}$ nanoparticles. Phys Proced 22: 63-27.

27. Hendraningrat L, Li S, Torseter O (2013) A coreflood investigation of nanofluid enhanced oil recovery. J Pet Sci Eng 111: 128-138. 